\title{
8
}

\section{DE LAS GUÍAS DE ESCRITURA AL MACRO-GÉNERO. LA RELEVANCIA DEL CONOCIMIENTO LINGÜÍSTICO EN LOS PROGRAMAS DE LECTURA Y ESCRITURA}

FROM THE WRITING GUIDES TO THE MACRO-GENRE. THE RELEVANCE OF LINGUISTIC KNOWLEDGE IN READING AND WRITING PROGRAMS

Martín M. Acebal

martinacebal@gmail.com / Universidad de Flores Universidad Nacional Guillermo Brown Argentina 


\section{RESUMEN}

El artículo desarrolla la eficacia de las nociones de género elemental y macrogénero para la intervención lingüístico-pedagógica sobre guías de escritura producidas en una asignatura universitaria para orientar a las y los estudiantes en la elaboración de textos. El trabajo reflexiona y muestra los resultados de una experiencia realizada en la asignatura «Prácticas Profesionales l» en el marco del Programa de Lectura y Escritura Académica (PROLEA) implementado en Universidad de Flores (Buenos Aires, Argentina). En el PROLEA, la o el especialista del Programa identifica las instancias de escritura de la asignatura y mantiene intercambios con la o el docente disciplinar para conocer sus objetivos y diseñar la intervención del Programa en la asignatura. En ocasiones nos encontramos con guías de escritura producidas por el/la docente disciplinar para la elaboración de los textos. El artículo sostiene y muestra con ejemplos que el abordaje lingüístico-genérico posibilita una relectura de estas guías desarrolladas en la asignatura, a la vez que permite diseñar su incorporación en las intervenciones didácticas del Programa. La capacidad explicativa de la propuesta genérica y los resultados obtenidos demuestran la importancia del especialista en el lenguaje, del andamiaje lingüístico-funcional y de la investigación en los Programas de Lectura y Escritura universitarios.

\section{PALABRAS CLAVE}

$>$ enseñanza de la escritura académica

$>$ guías de escritura

$>$ macro-género

$>$ programa de lectura y escritura

$>$ conciencia lingüística 


\section{ABSTRACT}

The article develops the effectiveness of the notions of elementary genre and macro-genre for the linguistic-pedagogical intervention on writing guides produced in a university subject to guide students in the elaboration of their texts. The work reflects and shows the results of an experience carried out in the subject «Professional Practices I» within the framework of the Academic Reading and Writing Program (PROLEA, in Spanish) implemented in the University of Flores (Buenos Aires, Argentina). In PROLEA the Program specialist identifies the writing instances of the subject and maintains exchanges with the disciplinary teacher to find out their objectives and design the Program intervention in the subject. Sometimes, the previous encounters and the intervention itself generate the first metalinguistic reflection in the teacher about the genres of the texts in her/his discipline. In others we find writing guides produced by the disciplinary teacher for the preparation of the texts. The article affirms and shows with examples that the linguistic-generic approach allows a rereading of these guides developed in the subject, at the same time that it allows to design their incorporation in the didactic interventions of the Program.

\section{KEYWORDS}

$>$ teaching academic writing

$>$ writing guides

$>$ macro-genre

$>$ reading and writing program

$>$ linguistic awareness 


\section{INTRODUCCIÓN}

Este trabajo comparte una experiencia implementada en el marco del Programa de Lectura y Escritura Académica (en adelante, PROLEA) que se desarrolla en la Universidad de Flores (Buenos Aires, Argentina). Presenta algunas estrategias para enseñanza de la escritura académica en la asignatura «Prácticas Profesionales I» del Profesorado Universitario en Actividad Física y Deporte y postula un modo de abordaje lingüístico de las guías de escritura presentes en la asignatura. Las guías de escritura constituyen instrumentos elaborados por la docente de manera previa a la intervención del PROLEA y sobre el cual el Programa debió trabajar para lograr la adecuación a su metodología de enseñanza de la escritura académica y su perspectiva lingüístico-genérica.

En tanto instrumento destinados a la facilitar la escritura en el ámbito de una asignatura - o del proceso de enseñanza que se realiza en una asignatura-, las guías conforman el espacio de encuentro entre los aspectos disciplinares — los contenidos de la asignatura, la organización y planificación de las clases, las estrategias didácticas, etc. - y los aspectos de la escritura académica —los vínculos entre escritura y aprendizaje, los géneros disciplinares, la conciencia lingüística de la o el docente de la asignatura, etc.-. Se trata, a la vez, de un encuentro documentado: la guía tiene la fuerza de la letra escrita, es un documento que instaura y registra la presencia de la escritura de las y los estudiantes en una asignatura.

En el marco de este artículo, las guías también incluyen una existencia en las aulas, en la interacción de docente y estudiantes. En este sentido, junto con su carácter de documento, la guía necesita ser considerada como un recurso que se articula de diferentes maneras con las actividades de enseñanza, puede inscribirse de un modo orgánico en estas actividades, o puede constituirse en un elemento marginal, sin ningún vínculo con el desarrollo de los contenidos o su evaluación.

El artículo tiene por objetivo desarrollar el trabajo de la integración de las guías desarrolladas por la docente de «Prácticas Profesionales I» al trabajo de intervención en la asignatura que realiza el docente del PROLEA. El énfasis estuvo puesto en la articulación de las guías con la noción de macro-género. Para esto comenzaremos con una revisión de las investigaciones actuales acerca de las guías de escritura en la enseñanza de la escritura académica. En un segundo momento propondremos el marco teórico lingüístico para el análisis de las guías, 
así como el marco pedagógico del PROLEA de la Universidad de Flores. En tercer lugar, ofreceremos una descripción de la perspectiva metodológica con la que se conformó el corpus de análisis. En cuarto lugar, se presentará el análisis de las guías a partir de la noción de macro-género. Finalmente, el artículo postulará una instancia de discusión con los trabajos relevados, en la que busca mostrar la eficacia del abordaje lingüístico en el uso de las guías para la enseñanza de la escritura.

DESARROLLO

\section{ANTECEDENTES}

Organizaremos los estudios que han abordado el uso de las guías de escritura en las tres problemáticas que consideramos transversales a las diferentes investigaciones: la relación de las guías de escritura con la enseñanza explícita de la escritura académica; el involucramiento de las guías de escritura en las actividades de enseñanza disciplinar; la consistencia teórica y genérica de las guías de escritura. Esto facilitará precisar la propuesta de este artículo y su discusión con los trabajos relevados.

\subsection{LAS GUÍAS DE ESCRITURA, SU RELACIÓN}

\section{CON LA ENSEÑANZA EXPLÍCITA DE LA ESCRITURA ACADÉMICA}

Diferentes investigaciones coinciden en la necesidad de inscribir a las guías en el marco de la enseñanza explícita de la escritura académica o universitaria (Ávila et. al., 2013; Moyano, 2011). En esta perspectiva, las guías forman parte de los soportes específicos -instrucciones, guiones, ayudas gráficas, entre otros (Jonassen \& Kim, 2010) — necesarios para la formación de escritoras y escritores, en especial de textos de carácter argumentativo (Larraín \& Burrows, 2020). Parte del aporte que realizan las guías de escritura reside en lograr explicitar las expectativas de las y los docentes, algo que ayuda a contrarrestar una de las principales dificultades que enfrentan las y los estudiantes en el nivel universitario (Hammer, 2017). Larraín \& Burrows (2020) sostienen que «una de las grandes 
razones por las que los estudiantes fallan en la escritura universitaria es porque [...] no tienen claridad acerca de lo que deben y se espera que escriban, y [...] esto se debe a la falta de claridad de los mismos docentes» (p.119). De la misma manera, Torres Perdigón (2018) sostiene que «la claridad de las explicaciones [en las guías] parece influir en una mejora de los estudiantes» (p.120). La claridad, en este estudio, se encuentra asociada a que las guías «especifiquen y aclaren lo que se busca con cada actividad en términos de lectura y escritura» (p.120).

Aunque en menor medida, también aparecen en estos trabajos referencias al rol de las guías de escritura en el aprendizaje del conocimiento disciplinar. Torres Perdigón (2018) menciona que las y los estudiantes «perciben un aporte en el conocimiento de la materia al poder leer y escribir con una guía y con pautas más específicas» (p.116). Sin embargo, esta misma autora atenúa esta afirmación al señalar que el análisis de los ensayos argumentativos producidos por las y los estudiantes muestra una mejora en la estructura y el uso de los marcadores, pero todavía presentan dificultades en la claridad de las tesis, así como en la pertinencia y la solidez de los argumentos.

\subsection{LAS GUÍAS DE ESCRITURA, SU INVOLUCRAMIENTO EN LAS ACTIVIDADES DE ENSEÑANZA DISCIPLINAR}

Una segunda problemática trata acerca de la inscripción de las guías en el proceso de enseñanza que se realiza en las asignaturas. En esta perspectiva, se identifican otras cuestiones relevantes. La primera de ellas alude a su carácter económico, de fácil incorporación por las y los docentes disciplinares que ofrecerían las guías. Larraín \& Burrows (2020) ven en esto una prueba de que «los y las docentes de asignatura sí pueden enseñar a escribir para aprender a través de dispositivos de bajo costo en términos de tiempo, como lo son las pautas o guías orientadoras» (p.123).

La segunda de las cuestiones alude a algo que Larrain \& Burrows (2020) formulan claramente: "la enseñanza de escritura argumentativa no se reduce a la entrega de pautas y guías» (p.120). Como señalan esta autora y este autor, hay consenso en la necesidad de apoyos complementarios como experiencias conjuntas de escritura (docente-estudiantes, estudiantes-estudiantes) (Bacha, 2010; Padilla, 2012), experiencias de análisis (Errázuriz, 2014; Padilla, 2012) 
y modelamiento (Bacha, 2010), entre otras estrategias. Casi en los mismos términos, Torres Perdigón (2018) menciona que en el trabajo con las guías «es indispensable la explicación de las pautas por parte del docente [..] no basta con enviar una suerte de instructivo: el profesor debe explicar y aclarar las guías, así como las expectativas frente a cada actividad» (p.116). Esto significa que más allá de su compromiso con una enseñanza explícita de la escritura académica, las guías - como dispositivo- necesitan insertarse en el contexto de las aulas, en el trabajo diario de las y los docentes, en su diseño de las clases. Como concluyen Ahern y Hernando (2020) en su investigación acerca de las guías para el Trabajo Final de Grado requerido para las carreras universitarias en España, «las Guías de Trabajo [...] son claramente insuficientes si no van acompañadas de tareas de análisis, lectura y escritura de los distintos apartados del TFG» (p.23). Las investigaciones coinciden también en que uno de los principales problemas que enfrenta el trabajo con guías de escritura reside en el lugar marginal o secundario que reciben en las clases. Romero González \& Álvarez Álvarez (2019) consignan que los pocos docentes que entregan materiales para aprender a escribir lo hacen sin ninguna explicación. Córdova (2009) refiere que algunos docentes que entregan pautas para la escritura luego reconocen que las y los estudiantes no las utilizan y que, de hecho, tampoco ellas y ellos las tienen en cuenta para la corrección. En un trabajo posterior, esta misma autora (Velázquez Rivera \& Córdova Jiménez, 2012) señala:

en cuanto a las pautas que se les entregan a los estudiantes, los profesores señalan —durante las entrevistas - que, si bien existen algunas referencias para ayudar a mejorar la escritura, estos aspectos terminan por descartarse, privilegiando el contenido conceptual (p.183).

El trabajo con las guías depende en gran medida de la consideración que tenga la o el docente de la importancia de la escritura en su asignatura. Como muestran las entrevistas realizadas por Torres Perdigón, el desinterés de las y los docentes puede deberse a que reproducen una distinción entre la «forma» 
y el "contenido» disciplinar; a que no se sienten competentes para hacerlo; o simplemente porque no disponen del tiempo necesario (2018, p.118). ${ }^{1}$

\subsection{LAS GUÍAS DE ESCRITURA, SU CONSISTENCIA TEÓRICA Y GENÉRICA}

La tercera problemática recurrente en los trabajos relevados se orienta a la precisión que poseen las guías de escritura. En algunos casos atribuyen a esta falta el bajo desempeño de las y los estudiantes (Hammer, 2017; Torres Perdigón, 2018), o la necesidad de desarrollar rediseños de las pautas y evaluar sus efectos (Larrain \& Burrows, 2020). En estos y otros trabajos, el análisis de las guías se focaliza, con diferentes énfasis, en tres grandes aspectos: la claridad y coherencia de las pautas; sus aspectos técnicos y retóricos; su consistencia teórica y genérica.

El primero de estos análisis es recurrente en muchas de las publicaciones. Las observaciones se encuentran estrechamente vinculadas con algo que mencionamos previamente: la demanda que se le hace a la guía de lectura por lograr explicitar las expectativas de la o el docente. Hammer (2017), en su trabajo acerca de las pautas de escritura de ensayos argumentativos en universidades de Australia, menciona que las y los estudiantes suelen recibir consejos vagos y conflictivos para la escritura del ensayo (Hammer, 2017, p.1070). Ahern y Hernando (2020), en el contexto español, coinciden al señalar que la falta de cohesión del Marco Teórico del Trabajo de Fin de Grado estaría determinada, en parte, por «las indicaciones de las Guías de Trabajo de Fin de Grado se revelan insuficientes e incluso contradictorias» (p.19). Larraín \& Burrows (2020), ya en el contexto chileno, llegan a conclusiones semejantes.

El segundo aspecto considerado alude a la relevancia que adquieren en las guías de escritura aspectos puramente técnicos o formales. En estos casos, los estudios no se detienen tanto en la vaguedad de las pautas o consejos, sino en el estable-

\footnotetext{
${ }^{1}$ Dice Torres Perdigón: «Es llamativa [...] una tensión: afirman la importancia de la forma de los textos de los estudiantes y de que estos reflejen el conocimiento disciplinar pero, al tiempo, piensan que el contenido puede desvincularse de la redacción. Es decir, se piensa que el aprendizaje puede estar desvinculado de las formas en la que se expresa. En algunos casos, es como si el contenido pudiese leerse «aparte» de su redacción, lo cual implica una concepción ni siquiera transversal, sino instrumental de la escritura. De los siete docentes, uno consideró en especial que este estudio añadió cuestiones a su curso que no le competían.» (2018, p.118).
} 
cimiento de precisiones que dejan fuera aspectos relevantes del texto que las y los estudiantes deben escribir. Romero González \& Álvarez Álvarez (2019) mencionan que las pautas dadas como iniciativa para la enseñanza de la escritura suelen estar orientadas «hacia aspectos más formales del texto: longitud [...], portada, índice, tamaño de letra, etc., sin llegar a abarcar otro tipo de cuestiones relacionadas con la estructura, los objetivos comunicativos o la audiencia del texto» (p.119). Larraín \& Burrows (2020) confirman las apreciaciones hechas por Lea \& Street (1998), que las guías dadas a las y los estudiantes tienen un carácter puramente técnico, reducido a un señalamiento de la estructura formal. Algo semejante identifican Ahern y Hernando (2020) en su análisis de las Guías de Trabajo de Fin de Grado vigente para el curso 2018-2019 en una universidad española. En este caso, las guías establecen «los apartados y subapartados obligatorios como algunas indicaciones acerca de los contenidos que deberá presentar cada uno de estos» (p.13).

El tercer aspecto alude a la consistencia teórica y genérica de las guías, es decir, al modo en que las guías precisan la pertenencia genérica de los textos que deben producir las y los estudiantes, así como de las particularidades de este género. Hammer (2017) identifica grandes deficiencias en los 30 documentos relevados en diferentes universidades australianas; los problemas recaen principalmente en una inadecuada concepción del ensayo argumentativo. Larraín \& Burrows (2020) retoman el trabajo de Hammer y en especial las pautas que esta autora brinda para la elaboración de guías de escritura de ensayo argumentativos, pero ponen un mayor énfasis en la identificación del género que realiza el documento analizado. En este sentido, afirman: «la Pauta 1 era ambigua respecto del género del texto. De hecho, en lugar de llamarse ensayo, se llamaba ficha de lectura» (p.121). Ahern \& Hernando (2020) mencionan que las guías brindadas describen la elaboración del Marco Teórico con una «revisión bibliográfica relacionada con la temática elegida», es decir que se confunden con lo que generalmente se conoce como un «estado de la cuestión», «estado del arte» o «antecedentes». Este último trabajo es de los pocos que, además de aludir a las imprecisiones genéricas de las guías, explicitan su concepción de género con una referencia a la obra de Martin \& Rose (2008).

Digamos, para concluir con esta revisión, que el estudio de las guías constituye un campo amplio y complejo, pero que puede ser pensado como atravesado por tres grandes problemáticas: su relación con la enseñanza explícita de la escritura académica, su involucramiento en las actividades de enseñanza disciplinar, su 
consistencia teórica y genérica. Los trabajos relevados aluden, en mayor o menor medida, a todos estos aspectos, para luego enfatizan algunos sobre otros. En este artículo también retomaremos estas tres áreas que conforman una suerte de topografía de un campo que es tanto de reflexión como de intervención. En relación con la primera de estas áreas, buscaremos posicionarnos acerca del rol que ocupan las guías en un programa de enseñanza explícita de la escritura académica en el interior de las asignaturas disciplinares, pero asignada a un especialista en la enseñanza de los géneros discursivos. En relación con la segunda de estas áreas, presentaremos las ventajas del trabajo conjunto entre el docente disciplinar y el especialista en el lenguaje, en tanto garantiza la efectiva incorporación de las guías de escritura dentro de las actividades de enseñanza. Por último, en relación con la demanda de un consistencia teórica y genérica de las guías de escritura, propondremos el aporte de las nociones de género elemental (Martin \& Rose, 2008) y macro-género (Martín, 1994), en tanto instrumentos para una adecuada descripción de los textos que las y los estudiantes deben producir.

\section{MARCO TEÓRICO Y PROPUESTA DIDÁCTICA}

\subsection{LA NOCIÓN DE GÉNERO Y MACRO-GÉNERO}

Para el abordaje de las guías de escritura analizadas, consideramos necesario una perspectiva como la que ofrece la Lingüística Sistémico Funcional que entienda al lenguaje como un recurso para la construcción de significados. En esta perspectiva, el género se concibe como una configuración recurrente de significados que ponen en acto prácticas sociales en una cultura dada (Martin \& Rose, 2008, p.6). El vínculo estrecho entre los géneros y las prácticas sociales demanda identificar cuál es el propósito —-social— que busca realizar esa práctica, cuáles son las etapas a través de las cuales alcanza ese propósito — que definen su «estructura esquemática» (Eggins, 1994) - y cuáles son los recursos lingüístico-discursivos que despliega para su realización. Tal como señalan Martin \& Rose (2008), esto también implica que el estudio de los géneros debe ir más allá de su abordaje individual, para poder considerar los modos en que los géneros, en una determinada cultura mantienen relaciones entre sí. 
Esta última formulación lleva a dos tipos de abordaje articulados. Por una parte, la posibilidad de identificar géneros elementales que mantienen relaciones entre sí dentro de una cierta cultura. Bathia (2002) sugiere pensarlos como una «constelación» de géneros próximos y, en algunos casos, superpuestos. Martin \& Rose (2008) proponen un abordaje sistemático de estas relaciones, las que nos permiten comprender, por ejemplo, el modo en que el discurso científico desarrolla diferentes géneros para la construcción de entidades y para la construcción de procesos; la sutileza en la caracterización estos géneros es lo que les permite a estos autores identificar géneros elementales y desarrollar sus vínculos sistemáticos. La propuesta de un vínculo estrecho con las prácticas combina el carácter convencionalizado que se asocia a la noción de género (Swales, 1990; Devitt, 2004), con su el carácter dinámico que tienen estas mismas prácticas (Berkenkotter \& Huckin, 1995).

Por otra parte, las relaciones entre los géneros pueden ocurrir de un modo simultáneo y permiten construir textos «más extensos que una página» (Martin, 1994, p.29). Esta coexistencia de géneros da cuenta de espacios sociales en que las prácticas han logrado tal grado de desarrollo y complejidad (Bajtin, 2008) que necesitan combinar estos géneros para constituir macro-géneros (Martin, 1994). La noción está estrechamente ligada al ámbito educativo —en el que se inscriben las guías que estamos estudiando- dado que sus condiciones favorecen la combinación de las unidades semánticas (Christie, 2002) o configuraciones recurrentes de significados (Martin \& Rose, 2008). En nuestro caso, los Informes de Observación requeridos a las y los estudiantes demandan una combinación muy compleja de géneros elementales, en tanto deben dar cuenta de un espacio social complejo, como el que constituye una institución educativa. El aporte más relevante de Martin (1994) consiste en valerse de las nociones de la gramática sistémico funcional (HaIliday, 1985) para identificar las diferentes estrategias ideacionales, interpersonales y textual con las que los textos devienen en macro-géneros, Entre las estrategias ideacionales — que retomaremos en este artículo-, Martin identifica a la «elaboración» (elaboration), la «extensión» (extension) y el «realce» (enhancement).

\subsection{LA PROPUESTA DIDÁCTICA DEL PROLEA}

El análisis y la experiencia que aquí referiremos se encuadra en el Programa de Lectura y Escritura Académicas (PROLEA) implementado en la Universidad de 
Flores. A los fines de caracterizar la propuesta, Moyano (2017) —diseñadora y directora de la propuesta — retoma la noción de «programa» de Schwalm (2002), quien la entiende como una serie de acciones con objetivos comunes, consistencia en sus métodos, procedimientos y evaluación. En este sentido, el PROLEA puede entenderse como un conjunto de acciones pedagógicas orientadas a la enseñanza de la lectura y la escritura de textos académicos y profesionales instalada de manera sistemática a lo largo de las diferentes carreras de la Universidad de Flores. En relación con sus objetivos, el PROLEA se propone, según Moyano (2017):

asistir a los estudiantes en esas tareas [lectura y escritura], guiándolos hasta que, al finalizar sus estudios de grado, puedan considerarse lectores y escritores con cierto grado de experticia, que hayan adquirido la habilidad de abordar un texto de manera efectiva, tanto en su lectura como su escritura y edición. (p.48)

De este modo, el programa postula sucesivas intervenciones en diferentes asignaturas a lo largo de las carreras de la Universidad de Flores. Las intervenciones son llevadas a cabo por una o un especialista en el lenguaje en un trabajo conjunto con el docente disciplinar. Este trabajo involucra una instancia de «negociación previa» (Moyano, 2010; Moyano \& Giudice, 2016) con la o el docente de la asignatura específica — que deviene así en profesor/a socio/a del Programa- a los fines de identificar y caracterizar las actividades de lectura y escritura académicas presentes en la asignatura. Esta propuesta pedagógico-didáctica está basada en las nociones de género y registro de la Lingüística Sistémico-Funcional (LSF) (Martin, 1997, 2001) y sus aplicaciones educativas (Rose \& Martin, 2012).

En términos metodológicos PROLEA desarrolla un dispositivo didáctico adaptable a diferentes niveles educativos (Moyano, 2007, 2010, 2011) y conformado por tres fases: i) deconstrucción del género, ii) escritura de un nuevo ejemplar del género, y iii) edición conjunta de los propios escritos. Cada una de estas etapas se encuentra permeada por una discusión sobre el contexto y el campo de pertenencia del texto, gracias a la interacción permanente con la/el docente de la asignatura específica. En ocasiones, los encuentros previos y la misma intervención generan la primera reflexión metalingüística en la o el docente acerca de las particularidades genéricas de los textos en su asignatura. En otros casos, como ocurre en la asignatura «Prácticas Profesionales I», el docente del Programa 
se encuentra con guías de escritura producidas por la o el docente disciplinar para la elaboración de los textos requeridos. En este artículo desarrollaremos el modo en que se articulan estos documentos con la perspectiva genérica y dispositivo didáctico del PROLEA.

\section{MARCO METODOLÓGICO}

Para el análisis que aquí se presenta se han tomado 24 producciones de estudiantes, realizadas durante los años 2016 y 2017 en la asignatura «Prácticas Profesionales I» (en el 2018 la docente realizó modificaciones en las guías). Los textos se consideran, en principio, como instanciaciones del macro-género estudiado. Decimos «en principio» porque este primer grupo de textos fue contrastado con la evaluación y los comentarios de la docente, de modo de identificar aquellos ejemplares que se adecuaran de mejor manera a las expectativas de la docente.

Este tipo de selección de textos forma parte de la etapa de Deconstrucción de un ejemplar modélico que requiere el dispositivo didáctico del PROLEA (Moyano, 2007, 2010, 2011). Habitualmente, la elección del texto modelo forma parte de la instancia de negociación con la o el docente disciplinar y concluye en la elección de un texto elaborado por un escritor experto. En ocasiones como la ocurrida en «Prácticas Profesionales I», el trabajo de deconstrucción debe tomar como ejemplares modélicos producciones de las o los estudiantes que hayan mostrado un buen desempeño. El análisis de estos textos permitió la descripción del macro-género y realizar su contrastación con las pautas establecidas en las guías

\section{ANÁLISIS Y RESULTADOS}

\subsection{LAS GUÍAS DE ESCRITURA EN LA ASIGNATURA}

La asignatura «Prácticas Profesionales I» pertenece al Profesorado Universitario en Actividad Física y Deporte de la Universidad de Flores. La asignatura se dicta a lo largo de todo el primer año de la carrera y destina ambos cuatrimestres a la 
elaboración de informes de observación de instituciones en las que se realiza la enseñanza de actividades físicas. El primer cuatrimestre se destina a la observación de una institución de enseñanza no formal y el segundo a una institución formal, por lo general una escuela primaria o secundaria.

En este artículo nos detendremos en la elaboración del Informe de Observación de la educación formal de la actividad física, en las guías brindadas por la docente de la asignatura y su incorporación dentro del trabajo realizado por el PROLEA. El Informe de Observación se organiza en tres etapas que constituyen tres sub-informes: un Diagnóstico Contextual, un Diagnóstico Institucional y un Diagnóstico de las Clases. Aunque en este artículo nos focalizaremos en el segundo de estos diagnósticos —el Institucional-corresponde hacer algunos comentarios acerca de la progresión genérica que conforman los tres informes. El pasaje del Diagnóstico Contextual al Diagnóstico de las Clases conlleva un análisis de los aspectos exteriores de la institución —ubicación espacial, características del barrio, movilidad, información demográfica, etc.- - a los específicamente vinculados con la enseñanza de la actividad física. En términos genéricos, esto supone un pasaje de géneros menos académicos - la descripción y la narración- hacia géneros académicos - tales como la explicación secuencial histórica, el informe descriptivo, composicional, entre otros- En el marco de una enseñanza de la escritura académica, esto brinda la posibilidad de trabajar las diferencias de entre los géneros de la vida cotidiana y los géneros que demanda el contexto universitario.

\subsection{LAS GUÍAS DE ESCRITURA COMO MACRO-GÉNERO}

En el 2016, al momento de comenzar el trabajo del PROLEA en la asignatura, la docente brindaba tres guías de escritura, una por cada uno de los «diagnósticos» - Contextual, Institucional, de las Clases-. Las guías — denominadas Fichascombinaban comentarios metodológicos para la elaboración del informe con pautas de escritura. Aquí nos focalizaremos en la Ficha 2, en la que se brindan las pautas para la escritura del Diagnóstico Institucional. Junto con las recomendaciones metodológicas, el documento incluía las siguientes pautas: 


\section{DATOS QUE DEBEN INTEGRAR EL DIAGNÓSTICO DEL ESTABLECIMIENTO EDUCATIVO. ASPECTO DINÁMICO}

$>$ Nombre de la Institución.

$>$ Breve reseña histórica (año de fundación, fundador, contrato fundacional).

$>$ Objetivos, fines, misión, visión (Datos del Proyecto Educativo Institucional).

$>$ Tipo de gestión.

$>$ Niveles y modalidades.

$>$ Planta funcional (personal directivo, docente, no docente, auxiliar, maestranza, preceptor, psicopedagogo, portero).

$>$ Alumnos (matrícula general).

$>$ Organigrama.

> Síntesis de los principales datos obtenidos a partir de documentos institucionales (reglamento de convivencia, $\mathrm{PEI}, \mathrm{PCl}$, etc.).

> Síntesis de los datos y reflexiones más relevantes aportadas por el/los entrevistado/s.

Como puede observarse, las pautas brindadas por el documento todavía se inscriben en el propósito metodológico de la Ficha. Aunque el texto abandona el género procedimental, se focaliza en la información que debe presentar el Informe -y que deberá reunir la o el estudiante-, sin brindar mayores precisiones acerca del modo en que esta información se constituye en un texto. Se reconocen así una serie de problemas:

$>$ Algunos de los puntos señalados no alcanzan a conformar un texto (por ejemplo, «Nombre de la institución»);

$>$ Se reúnen en un mismo punto elementos que refieren a géneros diferentes (por ejemplo, la reseña histórica y la caracterización del contrato fundacional);

$>$ Se discriminan elementos que pueden ser integrados en un mismo género (por ejemplo, organigrama, planta funcional, alumnos;

$>$ No especifica las jerárquicas ni semánticas entre los elementos.

En el marco del trabajo de intervención en la asignatura, se propuso caracterizar al Informe como un macro-género y realizar una descripción adecuada del mismo para poder diseñar una propuesta de enseñanza. Como ya se precisó en el apartado metodológico, en este caso se seleccionaron los textos producidos 
por las y los estudiantes con mejor calificación y valoración por parte de la docente. También se tuvieron en cuenta la evaluación y los comentarios realizados por la docente, incluso en aquellos textos con una baja calificación. El trabajo se complementó con sucesivos diálogos con la docente, destinados a precisar sus expectativas en relación con la actividad.

Todo este trabajo nos permitió reconocer en el Informe la composición de tres macro-género, correspondientes con lo que la docente califica como Diagnóstico Contextual, Diagnóstico Institucional y Diagnóstico de la Clases. Por una cuestión de extensión, en este artículo nos focalizaremos en la descripción del Aspecto Dinámico del Diagnóstico Institucional. Presentaremos el análisis en una tabla de tres columnas (Tabla 1).

La primera columna denominada «partes formales», y son las que se pueden reconstruir en parte por la guía dada por la docente y en parte por los subtítulos utilizados por las y los estudiantes en sus informes. La identificación de estas partes suele ser un componente recurrente en las guías de escritura y están destinadas a facilitar los apartados y subapartados que conforman el texto requerido. Sin embargo, como han señalado Ahern \& Hernando (2020), la falta de explicitación de los géneros en que se inscriben los textos de cada uno de los apartados termina por limitar la ayuda brindada por la enumeración de las partes formales.

La segunda columna identifica los géneros elementales (Martin \& Rose, 2008) que están involucrados en el macro-género del Aspecto Dinámico del Diagnóstico Institucional. Como puede reconocerse, se trata de la columna que establece los componentes genéricos del texto y que permite reunir las diferentes partes formales. Este aspecto es relevante porque nos permite pensar la existencia de desfasajes entre los géneros elementales y las partes formales, la comprensión de estos desfasajes es uno de los pasos para una integración adecuada de la información relevada en el proceso de observación. Más adelante desarrollaremos este aspecto con un ejemplo textual.

La tercera columna desarrolla las relaciones semánticas que se establecen entre los géneros elementales que conforman el macro-género. Aquí seguimos la propuesta de Martin (1994) para reconocer estrategias ideacionales, interpersonales y textuales en los macro-géneros; en este análisis nos focalizaremos en las primeras de estas estrategias. Estas relaciones son relevantes para evitar una atomización del texto, sea a través de sus partes formales o de sus géneros elementales. 


\begin{tabular}{|l|l|l|}
\hline \multicolumn{1}{|c|}{ Partes formales } & \multicolumn{1}{c|}{ Géneros elementales } & \multicolumn{1}{c|}{ Relaciones } \\
\hline Reseña histórica & [Explicación Secuencial Histórica] & \multicolumn{1}{c|}{ realce } \\
\hline Contrato fundacional & $\begin{array}{l}\text { [Informe descriptivo] Definición de } \\
\text { "contrato fundacional" } \\
\text { [Descripción] }\end{array}$ & $\begin{array}{l}\text { Relación de la } \\
\text { (consecuencia) con la Reseña } \\
\text { histórica. }\end{array}$ \\
\hline $\begin{array}{l}\text { Categorización (clasificación) de la } \\
\text { enseñanza y la institución (Formal, } \\
\text { No-formal, Informal; tipo de } \\
\text { gestión) }\end{array}$ & [Argumentación / Justificación] & $\begin{array}{l}\text { Relación de extensión con el } \\
\text { Contrato fundacional. }\end{array}$ \\
\hline $\begin{array}{l}\text { Niveles y modalidades Matrícula } \\
\text { Planta Funcional. Organigrama } \\
\text { Dimensión organizacional }\end{array}$ & [Descripción] & $\begin{array}{l}\text { Relación de extensión con la } \\
\text { Categorización }\end{array}$ \\
\hline
\end{tabular}

Tabla 1. Análisis del Aspecto Dinámico del Diagnóstico Institucional en términos de macro-género.

\subsection{LA REINSCRIPCIÓN DE UN GÉNERO ELEMENTAL EN UN MACRO-GÉNERO}

Uno de los principales aportes que ofrece la noción de macro-género es la de favorecer una mayor comprensión del modo en que se incorporan los textos en una unidad mayor. Para la producción de informes de observación esto es relevante en dos sentidos: en primer lugar, porque logra articular la información relevada — sea a través de la observación, de las entrevistas o los documentos ${ }^{2}$ - con su posterior textualización en el informe; en segundo lugar, porque logra anticipar la relación que tendrá la información textualizada en un género con pasajes anteriores y posteriores del mismo informe. Para mostrar esto, podemos analizar el modo en que se inscribe la narración acerca de la fundación de la institución observada en el Informe.

Tal como vimos, la descripción formal ofrecida por la guía dice «reseña histórica». Un abordaje más intuitivo de este elemento puede sugerir una secuencia de eventos que traten sobre la institución observada y en la que predominen las relaciones temporales. Sin embargo, en la medida en que inscribimos a este elemento del informe en el marco de macro-género, logramos anticipar el uso que se hará de

\footnotetext{
${ }^{2}$ Aunque no disponemos del espacio para un mayor desarrollo, corresponde señalar que uno de los aspectos que necesitó ser revisado en la guía de escritura es la homologación que realiza entre las partes formales y géneros elementales, con los «datos obtenidos a partir de documentos institucionales» y los «datos y reflexiones más relevantes aportadas por el/los entrevistado/s». Estas otras voces no se reúnen en apartados específicos, sino que se encuentran integrados a los diferentes géneros. Martin (1994, p.34) analiza esto como parte de la «estrategia de proyección» de los macro-géneros.
} 
la información que se brinde en esta «reseña histórica». Tal como hemos mostrado, el análisis de las producciones de las y los estudiantes, así como las observaciones de la docente, nos permitieron precisar que la narración requerida está orientada a constituirse, toda ella, en la causa del «contrato fundacional» que necesita ser descripto en el siguiente componente del Informe. La noción de «contrato fundacional» es retomada de Frigerio, Poggi y Tiramonti (1992) y es entendida como el mandato social que porta una institución, y para cuyo cumplimiento moviliza sus recursos. En este marco, se les demanda a las y los estudiantes que la «reseña histórica» permita identificar las causas para el surgimiento de ese mandato. De este modo se entiende que la secuencia de eventos que se presenta en el texto haga predominar las relaciones causales por sobre las temporales (Tabla 2).

\begin{abstract}
El Colegio Armenio Arzuni fue fundado en el año 1928, junto con la iglesia Armenia "Santa Cruz de Varak" que hoy forma parte de la institución. Sus fundadores fueron armenios que llegaron a Argentina y se asentaron en Bajo Flores, escapando del genocidio que sufrió su país entre los años 1915 y 1923 y constituyendo un barrio armenio de alrededor de 200 casas. Para poder realizar ambas construcciones previamente mencionadas, se conformó la primera comisión directiva presidida por Haroutium Manikian. (Texto 4)
\end{abstract}

El Colegio Armenio Arzuní ha sido un lugar de contención y aprendizaje para la comunidad armenia, teniendo como una de sus principales caracteristicas la búsqueda de integración desde sus comienzos hasta el dia de hoy, recibiendo alumnos y alumnas del resto de la sociedad sin distinción alguna. Cabe destacar que fue el primer colegio de la comunidad armenia en llevar a cabo esta inclusión, ya que las demás escuelas en su origen y primeros años sólo recibian alumnos de descendencia armenia. (Texto 4)
Explicación Secuencial Histórica de la fundación de la institución.

[causa]

Descripción del "contrato fundacional"

[efecto]

Tabla 2. Relaciones causales entre la Explicación Secuencial Histórica y la Descripción del contrato fundacional.

En el primer pasaje, la fundación del colegio se presenta como el evento o el acontecimiento que será explicado. La ubicación inicial del grupo nominal se reproduce en el siguiente párrafo, en el que se describe el mandato que estableció la institución al momento de su fundación. El propósito explicativo que tiene el primer pasaje se hace evidente en el modo en que se organizan los eventos, con el fenómeno a explicar — la fundación del colegio- en primer lugar. Martín \& 
Rose (2008) reconocen en esto una diferencia que mantienen las explicaciones con las recounts y accounts:

[Consequential explanation] differ from recount and accounts in that chronology is not used to organise texts. Rather, explanations are organised rhetorically, beginning with the event being explained and then unfolding through a set of relevant factors or consequences. (p.131)

En el primer fragmento, la organización retórica a la que aluden Martin y Rose se identifica en el ordenamiento de la secuencia de eventos, en donde la causalidad aparece invertida (primero la fundación, luego la llegada de sus fundadores a la Argentina, etc.). Esta organización es también la que permite establecer una continuidad entre los dos géneros al otorgarles a ambos un mismo punto de partida. El macro-género propone así una estrategia ideacional de «realce» (enhancement), en la que el género descriptivo califica de alguna manera la explicación secuencial histórica, al mostrar los efectos o consecuencias de esa secuencia de eventos en la misma institución. Los eventos, que en el género anterior se consideran involucrados en dar cuenta de la fundación de la institución, adquieren una nueva significación — forjar el mandato social del Colegio-, dada por la relación semántica establecida con el otro género.

El tratamiento de los eventos que realiza la Explicación Secuencial también forma parte de la transformación genérica que va del Diagnóstico Contextual al Diagnóstico de las Clases. Como hemos dicho, este recorrido también coincide con un aumento de la tecnicalidad y, fundamentalmente, de la presencia de géneros académicos en el Informe. En este sentido, el Diagnóstico Contextual también demanda entre sus partes formales una «breve reseña histórica del barrio o localidad ${ }^{3}{ }^{3}$ Sin embargo, en tales casos los textos hacen predominar

\footnotetext{
${ }^{3}$ Las partes formales mencionadas en la guías son las siguientes: ubicación geográfica del barrio o localidad; breve reseña histórica del barrio o localidad; mapa de la zona relevada con la ubicación exacta de la institución abordada; características de la población que la constituye; características de las calles y avenidas; tipos de transportes; tipos de comercios predominantes; instituciones (deportivas, culturales, sociales, educativas); tipos de viviendas; espacios verdes; problemas de mayor incidencia; principales necesidades que se perciben; fotos del lugar; síntesis de los datos y reflexiones más relevantes aportadas por los entrevistados del lugar.
} 
una organización temporal de los eventos, por lo que comienzan con el parcelamiento del territorio, los primeros asentamientos, hasta llegar a las condiciones actuales del barrio o la localidad. En este ejemplo se reconoce esta organización:

Almagro fue zona de quintas antes y después del paso del tren. Si bien el tren dio al barrio un giro fundamental, la evolución se afirmó como nunca a partir del tranvía. Pero también fue la fiebre amarilla la que terminó provocando un cambio profundo. Muchos porteños de clase alta abandonaron el centro de la Ciudad y se instalaron en sus quintas. Desde ese momento, nunca más volvieron al centro.

Hoy en día conocemos a este barrio como uno de los más tradicionales de la Ciudad y muy relacionado con el tango y los típicos cafés porteños. Por sus calles y bares circularon muchos de los grandes del tango y Almagro tiene el privilegio de ser el primer escenario que escuchó cantar a Carlos Gardel. (Diagnóstico Contextual. Texto 2)

En estos casos, el relato se encuentra más próximo a las organizaciones genéricas del sentido común, menos complejas y con una organización más lineal. Se reconoce una relación causal entre los eventos, pero sigue imponiéndose el orden temporal de los eventos.

El trabajo sobre los diferentes géneros que conforman el Diagnóstico Institucional —y los demás diagnósticos—, así como sus relaciones dentro de un macro-género, colabora a evitar algunas de las dificultades señaladas en las investigaciones sobre las guías de escritura. La focalización de la guía en la información que debe ser relevada no sólo deja fuera el modo en que debe ser textualizada por las y los estudiantes, también puede reproducir las diferencias entre el contenido disciplinar y su puesta en texto. De la misma manera, la consideración de los géneros involucrados en el Informe y de sus relaciones favorece la comprensión del modo particular en que la asignatura propone interpelar la información relevada a través de los diferentes instrumentos de recolección de datos propuestos. 


\section{DISCUSIÓN}

Como hemos propuesto en este artículo, las diferentes investigaciones acerca del uso de guías en la enseñanza de la escritura identifican tres grandes problemáticas: el modo en que las guías explicitan las expectativas de las y los docentes; el involucramiento de las guías en las actividades de enseñanza disciplinar; y la consistencia genérica de las guías. Los estudios han mostrado las problemáticas que despliegan en cada uno de estos ámbitos y las dificultades que surgen de la implementación de guías de escritura. En este trabajo hemos buscado mostrar que el trabajo que realiza el Programa de Lectura y Escritura Académica logra cubrir estas tres instancias por medio del trabajo conjunto entre el docente disciplinar y el especialista en el lenguaje que integra el Programa.

En relación con la identificación de las expectativas, hemos mostrado que la negociación previa que se estableció con la docente de la asignatura «Prácticas Profesionales I» permitió precisar qué es lo que ella esperaba de la actividad propuesta. En este sentido logramos caracterizar que la propuesta pedagógica se postula un gradual acercamiento a la institución educativa en la que se desarrolla la Educación Física. Este gradual acercamiento también supone una gradual elaboración de textos pertenecientes a géneros académicos en los que se pongan en texto las informaciones relevadas a través de los diferentes instrumentos de recolección de datos.

En la mayoría de las evidencias relevadas, la falta de explicitación de expectativas en las guías concluye en una reelaboración de ellas, a los fines de volver sus consignas lo más evidentes posibles. Esta propuesta se sustenta, en gran medida, sobre el postulado de que la enseñanza de la escritura quede a cargo de la o del docente disciplinar, ayudado o relevado, en gran medida, por lo señalado en el documento de la guía. Como también muestran las investigaciones, las pautas de escritura no son suficientes para la enseñanza de la escritura si no van acompañadas de actividades, tales como su explicación, ejercitación, mostración de ejemplos, etc. En este punto, varios estudios muestran que las y los docentes disciplinares declaran la falta de tiempo o de experticia para llevar adelante estas otras actividades. Ante esto, el PROLEA propuso que el trabajo de enseñanza explícita y el desarrollo de todas aquellas actividades complementarias a la guía de escritura estuvieran a cargo del especialista en el lenguaje que 
integra el Programa, con la asistencia y la colaboración de la docente disciplinar. No se trata entonces de recargar de tareas a esta docente ni de demandarle a un documento que sustituya un proceso de enseñanza explícita, sino de construir un trabajo asociado con el docente del Programa, de modo que el cronograma de la asignatura contemple entre sus actividades el desarrollo del dispositivo didáctico -Deconstrucción, Diseño, Construcción y Edición-necesario para complementar lo explicitado en la guía de escritura. Como afirma Moyano acerca de esta propuesta: «A diferencia de prácticas que simplemente ofrecen una consigna detallada a los estudiantes para que éstos resuelvan el problema planteado, en este modelo los docentes acompañan todo el proceso de aprendizaje, en un trabajo que se propone desde la heteronomía a la autonomía» $(2017$, p.56).

Finalmente, el presente artículo se focalizó en la tercera de las problemáticas acerca de las guías de escritura: su consistencia genérica. Este aspecto continúa, en cierta medida, lo desarrollado en los párrafos anteriores. Las limitaciones de las y los docentes disciplinares para dejar por escrito sus expectativas, así como sus reparos a asumir la tarea de la enseñanza explícita de la escritura académica, residen, en gran medida, en la falta de un metalenguaje que les permita una adecuada descripción de los textos esperados. En este sentido, los señalamientos acerca de la imprecisión genérica de las guías necesitan abordarse con una formación lingüístico-genérica y no necesariamente con la incorporación en las guías de definiciones acerca de lo que es, por ejemplo, una Explicación Secuencial Histórica. En el presente artículo se muestra el modo en que la noción de macro-género logra identificar no sólo los géneros que conforman los diferentes «diagnósticos» requeridos por la docente, sino también sus relaciones lógico-semánticas.

\section{CONCLUSIÓN}

A lo largo de este artículo buscamos mostrar que las guías de escritura pueden entenderse como dispositivos que involucran tres grandes dimensiones: su compromiso con la enseñanza de la escritura en el ámbito universitario; su efectiva incorporación dentro de las actividades de enseñanza y evaluación; y su consistencia teórica en términos disciplinares y genéricos. Las tres dimensiones interactúan 
de forma simultánea en los diferentes contextos de enseñanza que involucran el uso de guías de escritura. La diferenciación de estas dimensiones nos permitió identificar dónde han puesto su atención los trabajos que han abordado esta temática y cómo es posible que se generen discrepancias y tensiones entre las diferentes dimensiones en el marco del dictado de una asignatura universitaria. Sin embargo, lo más relevante en la identificación de estas instancias es reconocer todos los espacios que necesitan ser contemplados en la enseñanza explícita de la escritura académica. El PROLEA, por medio del trabajo articulado - pero no superpuesto- de la o del docente disciplinar y la o el lingüista logra cubrir estas diferentes instancias para, de este modo, potenciar el uso de las guías de escritura.

Las guías son, además, documentos para el acceso a las concepciones acerca de la escritura que tienen las y los docentes disciplinares (Bowen, 2009). Esto implica reconocer que algunas limitaciones que enfrentan las guías en su compromiso con la enseñanza de la escritura se deben a la relevancia que la o el docente le otorga a la escritura en su asignatura. Esta relevancia impactará tanto en el tiempo y la atención dedicada a las guías durante las clases, como en las precisiones genéricas ofrecidas en las pautas. Todo esto significa también que el trabajo con las guías se presenta como una instancia privilegiada para la construcción de una mayor conciencia lingüística en las y los docentes de las disciplinas. Este también es uno de los objetivos que debe perseguir un programa de enseñanza de la lectura y la escritura en el ámbito universitario.

\section{REFERENCIAS BIBLIOGRÁFICAS}

Ahern, A. y Hernando, A. (2020). Los trabajos de fin de grado en español y en inglés. Retos, y un intento de mejora, de la alfabetización académica en formación inicial de profesorado. Tendencias Pedagógicas, 36, 9-24.

Ávila, N., González-Álvarez, P., y Peñaloza, C. (2013). Creación de un programa de escritura en una universidad chilena: Estrategias para promover un cambio institucional. RMIE, 18(57), 537-560.

Bacha, N. (2010). Teaching the academic argument in a university EFL environment. Journal of English for Academic Purposes, 9(3), 229-241. 
Bajtin, M. (2008). Estética de la creación verbal. Buenos Aires: Siglo XXI.

Bathia, V. (2002). A generic view of academic discourse. En J. Flowerdew (Ed.), Academic discourse (pp.21-39). Cambridge: Cambridge University Press.

Berkenkotter, C. y T.N. Huckin (1995). Genre knowledge in disciplinary communication: cognition/culture/power. Hillsdale, NJ: Lawrence Erlbaum.

Bowen, G. (2009). Document Analysis as a Qualitative Research Method. Qualitative Research Journal, 9(2), 27-40.

Christie, F. (2002). Classroom Discourse Analysis: A functional perspective. Londres: Continuum.

Córdova Jiménez, A. (2009). La escritura en la formación académica en ciencias. Un estudio exploratorio acerca de las representaciones sociales de los profesores de la Licenciatura en Bioquímica. Tesis de Licenciatura en Lengua y Literatura Hispánica. Universidad Católica de Valparaíso, Chile.

Devitt, A. (2004). Writing genre. Carbonale: Southern Illinois University Press.

Eggins, S. (1994). An introduction to systemic functional linguistics. London: Cassell. Errázuriz, M.C. (2014). El desarrollo de la escritura argumentativa académica: los marcadores discursivos. Onomázein, 30, 217-236.

Frigerio, G., Poggi, M. y Tiramonti, G. (1992). Las instituciones educativas. Cara y ceca. Instrumentos para su comprensión. Buenos Aires: Troquel.

Halliday, M.A.K. (1985). Introduction to Functional Grammar. London: Edward Arnold. Hammer, S. (2017). An examination of the instruction provided in Australian essay guides for students' development of a critical viewpoint. Assess Eval High Edu, 42(7), 1069-1081.

Jonassen, D.H., y Kim, B. (2010). Arguing to learn and learning to argue: Design justifications and guidelines. Educational Technology Research and Development, 58, 439-457. Lea, M. y Street, B. (1998). Student writing in higher education: An academic literacies approach. Studies in Higher Education, 23, 157-172.

Martin, J.R. (1997). Analyzing genre: functional parameters. En F. Christie \& J.R. Martin (Eds). Genres and Institutions: Social Processes in the Workplace and School, (pp. 3-39). London: Continuum.

Martin, J.R. (2001). A context for genre: modelling social processes in functional linguistics. En J. Devilliers y R. Stainton (Eds.), Communication in Linguistics: papers in honour of Michael Gregory (pp.287-328). Toronto: GREF (Theoria Series 10).

Martin J.R. y Rose, D. (2008). Genre Relations. Mapping culture. London: Equinox. 
Martin J.R. y Rose, D. (2012). Learning to Write, Reading to Learn. Genre, Knowledge and Pedagogy in the Sydney School. UK: Equinox.

Moyano, E. (2007) Enseñanza de la lectura y la escritura basada en la teoría de género y registro de la LSF: resultados de una investigación. Revista Signos, 40(65), 573-608. Moyano, E. (2010). Escritura académica a lo largo de la carrera: un programa institucional, Revista Signos, 43(74), 465-488.

Moyano, E. (2011). Deconstrucción y edición conjuntas en la enseñanza de la escritura. La reflexión sobre género y discurso en la formación académica y profesional. En Anais VI Simpósio Internacional de Estudos de Gêneros Textuais (SIGET) [en línea]. Recuperado de: http://www.cchla.ufrn.br/visiget/

Moyano, E. (2017). Diseño e implementación de programas de lectura y escritura en el nivel universitario: principios y estrategias. Revista Lenguas Modernas, 50(2), 47-72. Moyano, E. y Giudice, J. (2016). Negotiation Between Professional Peers: Critical Strategy for a Reading and Writing Program at the University Level. Ilha do Desterro, 69(3), 157-172.

Núñez, J.A. y Errázuriz, C. (2020). Panoramas de la alfabetización académica en el ámbito iberoamericano: aportes para la calidad de la Educación Superior. Tendencias Pedagógicas, 36, 1-8.

Padilla, C. (2012). Escritura y argumentación académica: trayectorias estudiantiles, factores docentes y contextuales. magis, 5(10), 31-57.

Romero González, A.N. y Álvarez Álvarez, M. de las N. (2019). Representaciones sociales de los estudiantes universitarios de grado sobre la escritura académica. Íkala, 24(1), 103-118.

Swales, J. (1990). Genre analysis. English in academic and research settings. Cambridge: Cambridge University Press.

Schwalm, D. (2002). The Writing Program (Administrator) in Context: Where Am and I and Can I Still Behave Like a Faculty Member. En I. Ward \& W.J. Carpenter (Eds.), The Allyn \& Bacon Sourcebook for Writing Program Administrators (pp. 9-22). New York: Allyn \& Bacon.

Torres Perdigón, A. (2018). ¿Escritura disciplinar? Una experiencia a partir de la lectura crítica y la habilidad argumentativa en la universidad. RMIE, 23(76), 95-124.

Velásquez Rivera, M. y Córdova Jiménez, A. (2012). Representaciones sociales de profesores de un programa de Licenciatura en Bioquímica acerca de la enseñanza y aprendizaje de la escritura en la formación académica. Literatura y lingüística, 25, 169-191. 